\title{
A Unified Approach to Quinolizinium Cations and Related Systems by Ring- closing Metathesis
}

\author{
Ana Núñez, Ana M. Cuadro, Julio Alvarez-Builla and Juan J. Vaquero* \\ Departamento de Química Orgánica. \\ Universidad de Alcalá de Henares. \\ 28871-Alcalá de Henares. Madrid.
}

\section{Supporting Information}

(10 pages)

General experimental details. Melting points were uncorrected. Infrared spectra were recorded on $\mathrm{NaCl}$ pellets and spectral bands were reported in $\mathrm{cm}^{-1}$. ${ }^{1} \mathrm{H} \mathrm{NMR}$ and ${ }^{13} \mathrm{C}$ NMR spectra were recorded at $200 / 300 \mathrm{MHz}$ and $50 / 75 \mathrm{MHz}$ respectively. Chemical shifts were reported as $\delta$ values (ppm). The mass spectra (MS) were obtained as $\left(\mathrm{ESI}^{+}\right)$. Ruthenium's catalyst $\mathbf{1}$ and 2, 2-vinylpyridine and alcohols were purchased from Aldrich.

\section{General procedure for the preparation of salts 4.}

A solution of the corresponding alcohol $(2.6 \mathrm{mmol})$ and dry pyridine $(0.205 \mathrm{~g}, 2.6$ $\mathrm{mmol})$ in dry $\mathrm{CCl}_{4}(2 \mathrm{~mL})$ was stirred at room temperature for 5-10 min under argon. Then the mixture was added dropwise $(5-10 \mathrm{~min})$ over a cooled solution $\left(-10^{\circ} \mathrm{C}\right)$ of triflic anhydride $(0.733 \mathrm{~g}, 6 \mathrm{mmol})$ in dry $\mathrm{CCl}_{4}(3 \mathrm{~mL})$. The white solid formed was filtered off trough sodium sulphate, the solution was added via cannula to a solution of the vinyl derivative $(2 \mathrm{mmol})$ in dry $\mathrm{CCl}_{4}(2 \mathrm{~mL})$ and the reaction mixture stirred for 24 $\mathrm{h}$ at room temperature. The solvent was evaporated under reduced pressure and the residue purified by flash chromatography on silica gel (eluent: $\mathrm{CH}_{2} \mathrm{Cl}_{2} / \mathrm{MeOH}$ 9.5:0.5) or by washing with $\mathrm{Et}_{2} \mathrm{O}(\mathbf{4 b}, \mathbf{4 d}, \mathbf{4 i}$ and $\mathbf{4 j})$. 


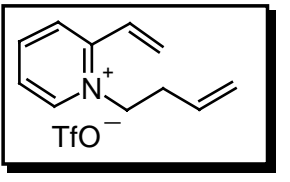

1-(3'-Butenyl)-2-vinylpyridinium triflate (4a). Following the general procedure, the reaction of 2-vinylpyridine $(0.21 \mathrm{~g}, 2 \mathrm{mmol})$ and 3-butenyl triflate $(0.53 \mathrm{~g}, 2.6 \mathrm{mmol})$, afforded $0.507 \mathrm{~g}(82 \%)$ of 4a as a pale-yellow oil: IR $(\mathrm{NaCl}) 3087,1621,1257,1512,1161,1030,785 \mathrm{~cm}^{-1} ;{ }^{1} \mathrm{H}$ NMR (300 MHz, CDCl $\left.)_{3}\right) \delta .92(\mathrm{~d}, 1 \mathrm{H}, J=6.2 \mathrm{~Hz}), 8.39(\mathrm{t}, 1 \mathrm{H}, J=7.9 \mathrm{~Hz}), 8.02(\mathrm{~d}$, $1 \mathrm{H}, J=8.2 \mathrm{~Hz}), 7.90(\mathrm{t}, 1 \mathrm{H}, J=6.4 \mathrm{~Hz}), 7.13(\mathrm{dd}, 1 \mathrm{H}, J=11.3,17.0 \mathrm{~Hz}) ; 6.28(\mathrm{dd}, 1 \mathrm{H}$, $J=1.3,17.0 \mathrm{~Hz}), 6.13(\mathrm{dd}, 1 \mathrm{H}, J=1.1,11.2 \mathrm{~Hz}), 5.84-5.71(\mathrm{~m}, 1 \mathrm{H}) ; 5.06(\mathrm{~d}, 1 \mathrm{H}, J=$ $10.2 \mathrm{~Hz}) ; 4.94(\mathrm{dd}, 1 \mathrm{H}, J=1.3,17.0 \mathrm{~Hz}) ; 4.79(\mathrm{t}, 2 \mathrm{H}, J=6.9 \mathrm{~Hz}) ; 2.65(\mathrm{dd}, 2 \mathrm{H}, J=6.9$, 14.1 Hz). ${ }^{13} \mathrm{C}$ NMR (75 MHz, acetone-d 6 ) $\delta 153.2,146.6,133.2,130.5,128.3,127.6$, 127.3, 119.7, 58.2, 34.7. MS $\left(\mathrm{ESI}^{+}\right) \mathrm{m} / \mathrm{z} 160\left(\mathrm{M}^{+}\right)$. Anal. Calcd. for $\mathrm{C}_{12} \mathrm{H}_{14} \mathrm{NSO}_{3} \mathrm{~F}_{3}$ : C, 46.60; H, 4.56; N, 4.53; S, 10.37. Found: C, 46.52; H, 4.79; N, 4.44; S, 10.41;

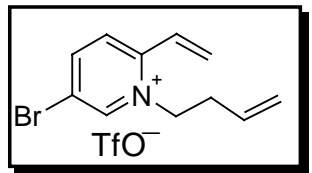

\section{5-Bromo-1-(3'-butenyl)-2-vinylpyridinium triflate (4b).}

Following the general procedure, the reaction of 5-bromo-2vinylpyridine $(0.368 \mathrm{~g}, 2 \mathrm{~mol})$ and 3-butenyl triflate $(0.53 \mathrm{~g}, 2.6$ mol) afforded $0.318 \mathrm{~g}(41 \%)$ of $\mathbf{4 b}$ as a white powder: $\mathrm{mp} 258-260^{\circ} \mathrm{C}\left(\mathrm{CH}_{2} \mathrm{Cl}_{2}: \mathrm{Et}_{2} \mathrm{O}\right)$. IR (NaCl) 3065, 1618, 1508, 1256, 1165, 1030, $759 \mathrm{~cm}^{-1} ;{ }^{1} \mathrm{H}$ NMR (300 MHz, $\left.\mathrm{CDCl}_{3}\right)$ $\delta 8.92(\mathrm{~d}, 1 \mathrm{H}, J=1.8 \mathrm{~Hz}), 8.45(\mathrm{dd}, 1 \mathrm{H}, J=1.8,8.8 \mathrm{~Hz}), 7.99(\mathrm{~d}, 1 \mathrm{H}, J=8.6 \mathrm{~Hz}), 7.10$ $(\mathrm{dd}, 1 \mathrm{H}, J=11.3,17.0 \mathrm{~Hz}) ; 6.35(\mathrm{~d}, 1 \mathrm{H}, J=17.0 \mathrm{~Hz}), 6.17$ (d, 1H, $J=11.3 \mathrm{~Hz}), 5.82-$ $5.71(\mathrm{~m}, 1 \mathrm{H}) ; 5.10(\mathrm{~d}, 1 \mathrm{H}, J=10.6 \mathrm{~Hz}) ; 4.96(\mathrm{~d}, 1 \mathrm{H}, J=15.9 \mathrm{~Hz}) ; 4.80(\mathrm{t}, 2 \mathrm{H}, J=6.9$ $\mathrm{Hz}) ; 2.65(\mathrm{dd}, 2 \mathrm{H}, J=6.9,14.1 \mathrm{~Hz}) .{ }^{13} \mathrm{C} \mathrm{NMR}\left(75 \mathrm{MHz}\right.$, acetone- $\left.\mathrm{d}_{6}\right) \delta 151.3,148.9$, 147.3, 133.1, 131.4, 128.4, 127.6, 121.4, 119.9, 58.8, 34.6. MS (ESI $\left.{ }^{+}\right) \mathrm{m} / \mathrm{z} 238\left(\mathrm{M}^{+}\right)$, $240(\mathrm{M}+2)$ Anal. Calcd. for $\mathrm{C}_{12} \mathrm{H}_{13} \mathrm{NBrSO}_{3} \mathrm{~F}_{3}: \mathrm{C}, 37.13 ; \mathrm{H}, 3.38 ; \mathrm{N}, 3.61 ; \mathrm{S}, 8.26$.

Found: C, 36.90; H, 3.45; N, 3.74; S, 8.16.

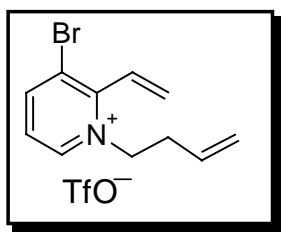

\section{3-Bromo-1-(3'-butenyl)-2-vinylpyridinium triflate (4c).} Following the general procedure, the reaction of 3-bromo-2vinylpyridine $(0.368 \mathrm{~g}, 2 \mathrm{mmol})$ and 3-butenyl triflate $(0.530 \mathrm{~g}, 2.6$ mmol) afforded $0.52 \mathrm{~g}(67 \%)$ of $\mathbf{4 c}$ as a pale-yellow oil: IR $(\mathrm{NaCl})$ 3084, 1463, 1261, 1156, 1030, $636 \mathrm{~cm}^{-1}$; ${ }^{1} \mathrm{H} \mathrm{NMR}\left(300 \mathrm{MHz}, \mathrm{CDCl}_{3}\right) \delta 9.07$ (d, 1H, $J=$ $6.0 \mathrm{~Hz}), 8.57(\mathrm{~d}, 1 \mathrm{H}, J=8.2 \mathrm{~Hz}), 7.87(\mathrm{dd}, 1 \mathrm{H}, J=6.2,8.4 \mathrm{~Hz}), 6.78(\mathrm{dd}, 1 \mathrm{H}, J=12.1$, $17.9 \mathrm{~Hz}) ; 6.22(\mathrm{~d}, 1 \mathrm{H}, J=12.1 \mathrm{~Hz}), 6.03(\mathrm{~d}, 1 \mathrm{H}, J=17.5 \mathrm{~Hz}), 5.83-5.69(\mathrm{~m}, 1 \mathrm{H}) ; 5.10$ 
$(\mathrm{d}, 1 \mathrm{H}, J=10.2 \mathrm{~Hz}) ; 5.00(\mathrm{~d}, 1 \mathrm{H}, J=17.2 \mathrm{~Hz}) ; 4.83(\mathrm{t}, 2 \mathrm{H}, J=7.1 \mathrm{~Hz}) ; 2.64(\mathrm{dd}, 2 \mathrm{H}, J$ $=6.9,14.1 \mathrm{~Hz}) .{ }^{13} \mathrm{C}$ NMR $\left(75 \mathrm{MHz}\right.$, acetone- $\left.\mathrm{d}_{6}\right) \delta 153.9,150.3,146.3,133.1,131.0$, 128.4, 127.8, 125.0, 119.7, 60.3, 34.6. $\mathrm{MS}\left(\mathrm{ESI}^{+}\right) \mathrm{m} / \mathrm{z} 238\left(\mathrm{M}^{+}\right), 240(\mathrm{M}+2)$. Anal. Calcd. for $\mathrm{C}_{12} \mathrm{H}_{13} \mathrm{NBrSO}_{3} \mathrm{~F}_{3}$ : C, 37.13; H, 3.38; N, 3.61; S, 8.26. Found: C, 37.24; H, $3.61 ; \mathrm{N}, 3.42 ; \mathrm{S}, 8.05$.

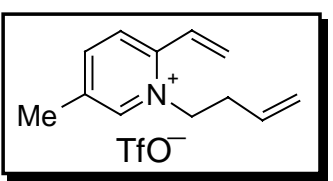

1-(3'-Butenyl)-5-methyl-2-vinylpyridinium triflate (4d).

Following the general procedure, the reaction of 3-methyl-6vinylpyridine $(0.238 \mathrm{~g}, 2 \mathrm{mmol})$ and 3-butenyl triflate $(0.53 \mathrm{~g}$, $2.6 \mathrm{mmol})$ afforded $0.388 \mathrm{~g}(60 \%)$ of $\mathbf{4 d}$ as a white powder: $\mathrm{mp} 60-62{ }^{\circ} \mathrm{C}\left(\mathrm{CH}_{2} \mathrm{Cl}_{2}\right.$ : $\left.\mathrm{Et}_{2} \mathrm{O}\right)$. IR ( $\left.\mathrm{NaCl}\right) 3076,1525,1250,1157,1030,850 \mathrm{~cm}^{-1} ;{ }^{1} \mathrm{H} \mathrm{NMR}\left(300 \mathrm{MHz}, \mathrm{CDCl}_{3}\right)$ $\delta 8.94(\mathrm{~s}, 1 \mathrm{H}) ; 8.49(\mathrm{~d}, 1 \mathrm{H}, J=7.9 \mathrm{~Hz}) ; 8.32(\mathrm{~d}, 1 \mathrm{H}, J=8.4 \mathrm{~Hz}) ; 7.43(\mathrm{dd}, 1 \mathrm{H}, J=11.2$, $17.0 \mathrm{~Hz}) ; 6.49$ (d, 1H, $J=17.0 \mathrm{~Hz}) ; 6.15(\mathrm{~d}, 1 \mathrm{H}, J=11.2 \mathrm{~Hz}) ; 5.99-5.85$ (m, 1H); 5.09$5.03(\mathrm{~m}, 2 \mathrm{H}) ; 4.90(\mathrm{t}, 2 \mathrm{H}, J=7.1 \mathrm{~Hz}) ; 2.83-2.76(\mathrm{~m}, 2 \mathrm{H}) ; 2.57(\mathrm{~s}, 3 \mathrm{H}) .{ }^{13} \mathrm{C}$ NMR $(75$ MHz, acetone- $\left.\mathrm{d}_{6}\right) \delta 150.7,147.2,145.9,138.9,133.4,129.6,128.1,126.9,119.6,58.2$, 34.9, 17.9. $\mathrm{MS}\left(\mathrm{ESI}^{+}\right) \mathrm{m} / \mathrm{z} 174\left(\mathrm{M}^{+}\right)$. Anal. Calcd. for $\mathrm{C}_{13} \mathrm{H}_{16} \mathrm{NSO}_{3} \mathrm{~F}_{3}: \mathrm{C}, 48.29 ; \mathrm{H}, 4.99$; N, 4.33; S, 9.92. Found: C, 48.57; H, 4.61; N, 4.43; S, 9.71.

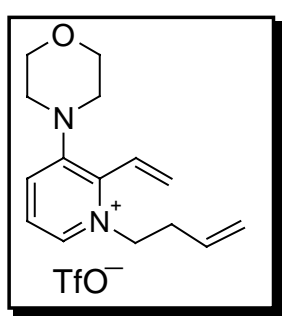

\section{1-(3'-Butenyl)-3-morpholin-4-yl-2-vinylpyridinium triflate (4e).}

Following the general procedure, the reaction of 3-morpholin-4-yl-2vinylpyridine $(0.38 \mathrm{~g}, 2 \mathrm{mmol})$ and 3-butenyltriflate $(0.53 \mathrm{~g}, 2.6$ mmol) afforded $0.433 \mathrm{~g}(55 \%)$ of $\mathbf{4 e}$ as a yellow oil: $\mathrm{IR}(\mathrm{NaCl}) 2854$, 1578, 1449, 1260, 1154, 1030, $755 \mathrm{~cm}^{-1} ;{ }^{1} \mathrm{H}$ NMR $(300 \mathrm{MHz}$, acetone- $\left._{6}\right) \delta 8.65(\mathrm{dd}, 1 \mathrm{H}, J=1.0,6.1 \mathrm{~Hz}), 8.23(\mathrm{dd}, 1 \mathrm{H}, J=1.0,8.4 \mathrm{~Hz}), 7.96(\mathrm{dd}$, $1 \mathrm{H}, J=6.0,8.4 \mathrm{~Hz}), 7.13(\mathrm{dd}, 1 \mathrm{H}, J=12.0,17.9 \mathrm{~Hz}) ; 6.29(\mathrm{dd}, 1 \mathrm{H}, J=1.3,17.9 \mathrm{~Hz})$, $6.19(\mathrm{dd}, 1 \mathrm{H}, J=1.0,12.0 \mathrm{~Hz}), 6.00-5.79(\mathrm{~m}, 1 \mathrm{H}) ; 5.13-5.10(\mathrm{~m}, 1 \mathrm{H}) ; 5.04-5.02(\mathrm{~m}$, $1 \mathrm{H}) ; 4.81(\mathrm{t}, 2 \mathrm{H}, J=7.2 \mathrm{~Hz}) ; 3.74(\mathrm{t}, 4 \mathrm{H}, J=4.6 \mathrm{~Hz}) ; 3.18(\mathrm{t}, 4 \mathrm{H}, J=4.8 \mathrm{~Hz}) ; 2.76$ $(\mathrm{dd}, 2 \mathrm{H}, J=7.2,14.3 \mathrm{~Hz}) .{ }^{13} \mathrm{C} \mathrm{NMR}\left(50 \mathrm{MHz}, \mathrm{CDCl}_{3}\right) \delta 151.4,146.5,138.0,134.1$, 131.4, 128.2, 126.6, 125.8, 119.8, 66.2, 58.3, 50.9, 34.3. MS (ESI $\left.{ }^{+}\right) \mathrm{m} / \mathrm{z} 245\left(\mathrm{M}^{+}\right)$. Anal. Calcd. for $\mathrm{C}_{16} \mathrm{H}_{21} \mathrm{~N}_{2} \mathrm{SO}_{4} \mathrm{~F}_{3}: \mathrm{C}, 48.72 ; \mathrm{H}, 5.37 ; \mathrm{N}, 7.10 ; \mathrm{S}, 8.13$. Found: C, 48.54; H, $5.26 ; \mathrm{N}, 7.01 ; \mathrm{S}, 8.45$. 


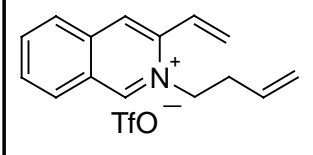

1-(3'-Butenyl)-3-vinylisoquinolinium triflate (4f). Following the general procedure, the reaction of 3-vinylisoquinoline $(0.31 \mathrm{~g}$, $2 \mathrm{mmol})$ and 3-butenyl triflate $(0.530 \mathrm{~g}, 2.6 \mathrm{mmol})$ afforded $0.395 \mathrm{~g}(55 \%)$ of $\mathbf{4 f}$ as a yellow powder: $\mathrm{mp} 91-93^{\circ} \mathrm{C}$, IR $(\mathrm{NaCl}) 3049,1643,1403$, 1257, 1156, 1029, $757 \mathrm{~cm}^{-1} ;{ }^{1} \mathrm{H}$ NMR $\left(300 \mathrm{MHz}, \mathrm{CDCl}_{3}\right) \delta 10.21(\mathrm{~s}, 1 \mathrm{H}), 8.64$ (d, 1H, $J$ $=8.4 \mathrm{~Hz}), 8.19(\mathrm{~s}, 1 \mathrm{H}), 8.13-8.05(\mathrm{~m}, 2 \mathrm{H}), 7.93(\mathrm{t}, 1 \mathrm{H}, J=7.1 \mathrm{~Hz}) ; 7.09(\mathrm{dd}, 1 \mathrm{H}, J=$ 11.3, $17.0 \mathrm{~Hz}) ; 6.18(\mathrm{~d}, 1 \mathrm{H}, J=17.0 \mathrm{~Hz}), 6.00(\mathrm{~d}, 1 \mathrm{H}, J=11.2 \mathrm{~Hz}), 5.94-5.82(\mathrm{~m}, 1 \mathrm{H})$; $5.05(\mathrm{~d}, 1 \mathrm{H}, J=9.9 \mathrm{~Hz}) ; 4.99-4.89(\mathrm{~m}, 3 \mathrm{H}) ; 2.74(\mathrm{dd}, 2 \mathrm{H}, J 6.9,13.9 \mathrm{~Hz}) .{ }^{13} \mathrm{C}$ NMR $(75$ MHz, acetone- $\left.\mathrm{d}_{6}\right) \delta 151.5,144.1,138.9,137.7,133.1,131.6,130.7,128.3,127.9,127.5$, 127.1, 124.9, 119.5, 58.2, 34.6. $\mathrm{MS}\left(\mathrm{ESI}^{+}\right) \mathrm{m} / \mathrm{z} 210\left(\mathrm{M}^{+}\right)$. Anal. Calcd. for $\mathrm{C}_{16} \mathrm{H}_{16} \mathrm{NSO}_{3} \mathrm{~F}_{3}$ : C, 53.48; H, 4.49; N, 3.90; S, 8.92. Found: C, 53.61; H, 4.30; N, 4.02; S, 8.78.

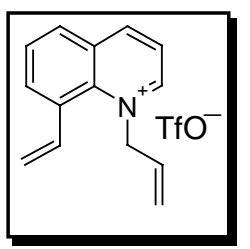

1-(2'-Propenyl)-8-vinylquinolinium triflate $(\mathbf{4 g})$. Following the general procedure, the reaction of 8 -vinylquinoline $(0.31 \mathrm{~g}, 2 \mathrm{mmol})$ and allyl triflate $(0.494 \mathrm{~g}, 2.6 \mathrm{mmol})$ afforded $0.153 \mathrm{~g}(47 \%)$ of $\mathbf{4 g}$ as a yellow oil: IR $(\mathrm{NaCl}) 3084,1529,1259,1157,1030,768 \mathrm{~cm}^{-1} ;{ }^{1} \mathrm{H}$ NMR (300 MHz, acetone-d $)) \delta 9.50(\mathrm{dd}, 1 \mathrm{H}, J=1.3,5.8 \mathrm{~Hz}), 9.40(\mathrm{dd}, 1 \mathrm{H}, J=1.3,8.4$ $\mathrm{Hz}), 8.48(\mathrm{dd}, 1 \mathrm{H}, J=1.3,8.2 \mathrm{~Hz}), 8.27(\mathrm{dd}, 1 \mathrm{H}, J=6.0,8.2 \mathrm{~Hz}), 8.16(\mathrm{~d}, 1 \mathrm{H}, J=7.3$ Hz); 8.05 (t, 1H, $J=7.9 \mathrm{~Hz}) ; 7.63$ (dd, 1H, $J=10.8,17.0 \mathrm{~Hz}), 6,43-6.31$ (m, 1H), 6.01$5.99(\mathrm{~m}, 2 \mathrm{H}) ; 5.82(\mathrm{dd}, 1 \mathrm{H}, J=0.9,17.0 \mathrm{~Hz}) ; 5.71(\mathrm{dd}, 1 \mathrm{H}, J=0.9,10.8 \mathrm{~Hz}) ; 5.48(\mathrm{dt}$, $1 \mathrm{H}, J=1.5,9.1 \mathrm{~Hz}) ; 5.16(\mathrm{dt}, 1 \mathrm{H}, J=1.6,17.3 \mathrm{~Hz}) .{ }^{13} \mathrm{C}$ NMR $\left(75 \mathrm{MHz}\right.$, acetone- $\left.\mathrm{d}_{6}\right) \delta$ $152.5,149.8,139.2,138.0,136.6,133.1,132.6,132.3,131.9,130.5,122.9,121.1$, 120.2, 63,1. MS $\left(\mathrm{ESI}^{+}\right) \mathrm{m} / \mathrm{z} 196\left(\mathrm{M}^{+}\right)$. Anal. Calcd. for $\mathrm{C}_{15} \mathrm{H}_{14} \mathrm{NSO}_{3} \mathrm{~F}_{3}: \mathrm{C}, 52.17 ; \mathrm{H}$, 4.09; N, 4.06; S, 9.28. Found: C, 52.30; H, 4.09; N, 4.27; S, 9.14.

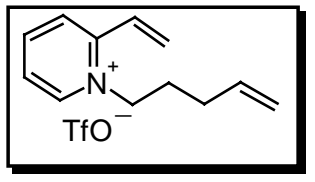

1-(4'-Pentenyl)-2-vinylpyridinium triflate (4h). Following the general procedure, the reaction of 2-vinylpyridine $(0.21 \mathrm{~g}, 2.0$ $\mathrm{mmol})$ and 4-pentenyl triflate $(0.567 \mathrm{~g}, 2.6 \mathrm{mmol})$ afforded 0.452 $\mathrm{g}(70 \%)$ of $\mathbf{4 h}$ as a pale-yellow oil: IR $(\mathrm{NaCl}) 3085,1512,1621,1257,1157,1030,787$ $\mathrm{cm}^{-1} ;{ }^{1} \mathrm{H}$ NMR $\left(300 \mathrm{MHz}, \mathrm{CDCl}_{3}\right) \delta 8.92(\mathrm{~d}, 1 \mathrm{H}, J=6.2 \mathrm{~Hz}), 8.43(\mathrm{t}, 1 \mathrm{H}, J=7.9 \mathrm{~Hz})$, $8.10(\mathrm{~d}, 1 \mathrm{H}, J=8.0 \mathrm{~Hz}), 7.90(\mathrm{t}, 1 \mathrm{H}, J=7.5 \mathrm{~Hz}), 7.08(\mathrm{dd}, 1 \mathrm{H}, J=11.3,17.0 \mathrm{~Hz}) ; 6.32$ 
(d, 1H, $J=17.0 \mathrm{~Hz}), 6.11(\mathrm{~d}, 1 \mathrm{H}, J=11.3 \mathrm{~Hz}), 5.79-5.65(\mathrm{~m}, 1 \mathrm{H}) ; 5.07-4.99(\mathrm{~m}, 2 \mathrm{H})$; $4.63(\mathrm{t}, 2 \mathrm{H}, J=7.7 \mathrm{~Hz}) ; 2.19-2.12(\mathrm{~m}, 2 \mathrm{H}) ; 2.02-1.86(\mathrm{~m}, 2 \mathrm{H}) .{ }^{13} \mathrm{C}$ NMR $(75 \mathrm{MHz}$, acetone- $\left.\mathrm{d}_{6}\right) \delta 153.5,146.6,146.5,137.5,130.5,128.3,127.8,116.4,59.0,54.9,30.7$. MS $\left(\mathrm{ESI}^{+}\right) \mathrm{m} / \mathrm{z} 174\left(\mathrm{M}^{+}\right)$. Anal. Calcd. for $\mathrm{C}_{13} \mathrm{H}_{16} \mathrm{NSO}_{3} \mathrm{~F}_{3}: \mathrm{C}, 48.29 ; \mathrm{H}, 4.99 ; \mathrm{N}, 4.33$; S, 9.92. Found: C, 48.59; H, 5.17; N, 4.12; S, 10.11.

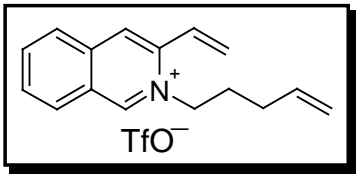

1-(4'-Pentenyl)-3-vinylisoquinolinium triflate (4i). Following the general procedure, the reaction of 3-vinylisoquinoline $(0.31$ $\mathrm{g}, 2 \mathrm{mmol})$ and 4-pentenyl triflate $(0.567 \mathrm{~g}, 2.6 \mathrm{mmol})$ afforded $0.336 \mathrm{~g}(45 \%)$ of $\mathbf{4 i}$ as a yellow powder: $\mathrm{mp} 104-105^{\circ} \mathrm{C}$. IR $(\mathrm{NaCl}) 3052,1643,1514$, 1258, 1156, 1029, $768 \mathrm{~cm}^{-1} ;{ }^{1} \mathrm{H}$ NMR $\left(200 \mathrm{MHz}, \mathrm{CDCl}_{3}\right) \delta 10.12(\mathrm{~s}, 1 \mathrm{H}), 8.69(\mathrm{~s}, 1 \mathrm{H})$; $8.53(\mathrm{~d}, 1 \mathrm{H}, J=8.4 \mathrm{~Hz}), 8.36(\mathrm{~d}, 1 \mathrm{H}, J=8.4 \mathrm{~Hz}), 8.24(\mathrm{t}, 1 \mathrm{H}, J=6.9 \mathrm{~Hz}), 8.03(\mathrm{t}, 1 \mathrm{H}, J$ $=7.1 \mathrm{~Hz}) ; 7.45(\mathrm{dd}, 1 \mathrm{H}, J=11.2,17.0 \mathrm{~Hz}) ; 6.43(\mathrm{~d}, 1 \mathrm{H}, J=16.8 \mathrm{~Hz}), 6.02(\mathrm{~d}, 1 \mathrm{H}, J=$ $11.2 \mathrm{~Hz}), 5.30-5.80(\mathrm{~m}, 1 \mathrm{H}) ; 5.08(\mathrm{~d}, 1 \mathrm{H}, J=15.7 \mathrm{~Hz}) ; 5.01-4.96(\mathrm{~m}, 3 \mathrm{H}) ; 2.31-2.16$ $(\mathrm{m}, 4 \mathrm{H}) ; 2.17-2.06(\mathrm{~m}, 2 \mathrm{H}) .{ }^{13} \mathrm{C}$ NMR $\left(75 \mathrm{MHz}\right.$, acetone-d $\left.{ }_{6}\right) \delta 151.5,144.2,139.1$, $137.7,137.5,131.7,130.8,128.3,127.9,127.8,126.9,124.9,116.1,58.9,30.6,29.9$. MS $\left(\mathrm{ESI}^{+}\right) \mathrm{m} / \mathrm{z} 224\left(\mathrm{M}^{+}\right)$. Anal. Calcd. for $\mathrm{C}_{17} \mathrm{H}_{18} \mathrm{NSO}_{3} \mathrm{~F}_{3}$ : C, 54.68; H, 4.86; N, 3.75; S, 8.59. Found: C, 54.32; H, 4.73; N, 3.88; S, 8.40.

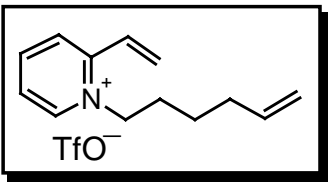

1-(5'-Hexenyl)-2-vinylpyridinium triflate $(4 \mathbf{j})$. Following the general procedure, the reaction of 2-vinylpyridine $(0.21 \mathrm{~g}, 2$ $\mathrm{mmol})$ and 5-hexenyl triflate $(0.603 \mathrm{~g}, 2.6 \mathrm{mmol})$ afforded $0.66 \mathrm{~g}$ $(98 \%)$ of $\mathbf{4} \mathbf{j}$ as a pale-yellow oil: IR $(\mathrm{NaCl}) 3084,1622,1511,1257,1158,1029,786$ $\mathrm{cm}^{-1} ;{ }^{1} \mathrm{H}$ NMR $\left(300 \mathrm{MHz}, \mathrm{CDCl}_{3}\right) \delta 9.00(\mathrm{~d}, 1 \mathrm{H}, J=6.1 \mathrm{~Hz}), 8.41(\mathrm{t}, 1 \mathrm{H}, J=7.7 \mathrm{~Hz})$, $8.03(\mathrm{~d}, 1 \mathrm{H}, J=7.9 \mathrm{~Hz}), 7.94(\mathrm{td}, 1 \mathrm{H}, J=1.3,7.7 \mathrm{~Hz}), 7.09(\mathrm{dd}, 1 \mathrm{H}, J=11.3,17.2 \mathrm{~Hz})$; $6.29(\mathrm{~d}, 1 \mathrm{H}, J=17.0 \mathrm{~Hz}), 6.12(\mathrm{~d}, 1 \mathrm{H}, J=11.3 \mathrm{~Hz}), 5.82-5.62(\mathrm{~m}, 1 \mathrm{H}) ; 5.05-4.95(\mathrm{~m}$, $2 \mathrm{H}) ; 4.68$ (t, 2H, $J=7.9 \mathrm{~Hz}) ; 2.16-2.05(\mathrm{~m}, 2 \mathrm{H}) ; 1.98-1.82(\mathrm{~m}, 2 \mathrm{H}) ; 1.66-1.43(\mathrm{~m}, 2 \mathrm{H})$. ${ }^{13} \mathrm{C}$ NMR (75 MHz, acetone- $\left.\mathrm{d}_{6}\right) \delta 146.4,146.3,138.6,130.3,128.2,127.6,127.5$, 115.4, 59.2, 33.6, 30.4, 25.8. MS (ESI $\left.{ }^{+}\right) \mathrm{m} / \mathrm{z} 188\left(\mathrm{M}^{+}\right)$. Anal. Calcd. for $\mathrm{C}_{14} \mathrm{H}_{18} \mathrm{NSO}_{3} \mathrm{~F}_{3}$ : C, 49.84; H, 5.38; N, 4.15; S, 9.50. Found: C, 49.72; H, 5.17; N, 4.41; S, 9.73. 


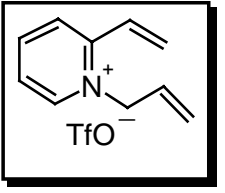

$N$-Allyl-2-vinylpyridinium triflate (4k). Following the general procedure, the reaction of 2-vinylpyridine $(0.21 \mathrm{~g}, 2 \mathrm{mmol})$ and allyl triflate $(0.494 \mathrm{~g}, 2.6 \mathrm{mmol})$ afforded $0.348 \mathrm{~g}(59 \%)$ of $\mathbf{4 k}$ as a paleyellow oil: IR (NaCl) 3090, 1621, 1508, 1258, 1158, 1029, $786 \mathrm{~cm}^{-1} ;{ }^{1} \mathrm{H}$ NMR (200 $\left.\mathrm{MHz}, \mathrm{CDCl}_{3}\right) \delta 8.94(\mathrm{dd}, 1 \mathrm{H}, J=1.0,6.4 \mathrm{~Hz}), 8.45(\mathrm{td}, 1 \mathrm{H}, J=1.0,7.9 \mathrm{~Hz}), 8.06(\mathrm{~d}$, $1 \mathrm{H}, J=6.9 \mathrm{~Hz}), 7.96(\mathrm{td}, 1 \mathrm{H}, J=1.4,7.7 \mathrm{~Hz}), 7.07(\mathrm{dd}, 1 \mathrm{H}, J=11.3,17.2 \mathrm{~Hz}) ; 6.28(\mathrm{~d}$, $1 \mathrm{H}, J=17.0 \mathrm{~Hz}), 6.11(\mathrm{~d}, 1 \mathrm{H}, J=11.3 \mathrm{~Hz}), 6.11-5.94(\mathrm{~m}, 1 \mathrm{H}) ; 5.46(\mathrm{dt}, 1 \mathrm{H}, J=1.3$, $10.2 \mathrm{~Hz}) ; 5.34-5.28(\mathrm{~m}, 2 \mathrm{H}) ; 5.20(\mathrm{dt}, 1 \mathrm{H}, J=1.5,17.2 \mathrm{~Hz}) .{ }^{13} \mathrm{C}$ NMR $(75 \mathrm{MHz}$, acetone- $\left.\mathrm{d}_{6}\right) \delta 153.4,146.8,146.2,131.2,130.3,128.0,127.7,127.4,120.9,60.8$. MS $\left(\mathrm{ESI}^{+}\right) \mathrm{m} / \mathrm{z} 146\left(\mathrm{M}^{+}\right)$. Anal. Calcd. for $\mathrm{C}_{11} \mathrm{H}_{12} \mathrm{NSO}_{3} \mathrm{~F}_{3}: \mathrm{C}, 44.74 ; \mathrm{H}, 4.10 ; \mathrm{N}, 4.74 ; \mathrm{S}$, 10.86. Found: C, 44.52; H, 4.20; N, 4.89; S, 10.63 .

\section{Ring-closing metathesis of salts 4. General procedure for cations 3a-g.}

To a solution of the corresponding salt $4(0.2 \mathrm{mmol})$ in dry $\mathrm{CH}_{2} \mathrm{Cl}_{2}(1.5 \mathrm{~mL})$, ruthenium catalyst $1(2 \mathrm{~mol} \%)$ for $\mathbf{3 a}$ or $\mathbf{2}(5 \mathrm{~mol} \%)$ for $\mathbf{3 b}-\mathbf{g}$ in $\mathrm{CH}_{2} \mathrm{Cl}_{2}(1 \mathrm{~mL})$ was added under argon atmosphere. The reaction mixture was stirred for 1-3 $\mathrm{h}$ at room temperature.

Then, the solvent was evaporated under reduced pressure and the residue purified by flash chromatography (eluent: $\mathrm{CH}_{2} \mathrm{Cl}_{2} / \mathrm{MeOH}$ 9.3:0.7) or by washing with $\mathrm{CH}_{2} \mathrm{Cl}_{2}: \mathrm{Et}_{2} \mathrm{O}$ (3g).

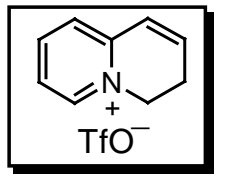

3,4-Dihydroquinolizinium triflate (3a). Following the general procedure, after stirring for $1.5 \mathrm{~h}, 46.8 \mathrm{mg}(83 \%)$ of $\mathbf{3 a}$ were obtained as a grey powder: mp $124-125^{\circ} \mathrm{C}\left(\right.$ Acetone: $\left.\mathrm{Et}_{2} \mathrm{O}\right) . \mathrm{IR}(\mathrm{NaCl}) 3086,1646$, 1507, 1263, 1150, 1031, $808 \mathrm{~cm}^{-1} ;{ }^{1} \mathrm{H}$ NMR (300 MHz, $\left.\mathrm{CDCl}_{3}\right) \delta 8.92(\mathrm{~d}, 1 \mathrm{H}, J=6.2$ $\mathrm{Hz}) ; 8.33(\mathrm{t}, 1 \mathrm{H}, J=7.8 \mathrm{~Hz}) ; 7.80(\mathrm{t}, 1 \mathrm{H}, J=7.1 \mathrm{~Hz}) ; 7.65(\mathrm{~d}, 1 \mathrm{H}, J=7.9 \mathrm{~Hz}) ; 6.89$ $6.83(\mathrm{~m}, 1 \mathrm{H}) ; 6.72(\mathrm{~d}, 1 \mathrm{H}, J=9.7 \mathrm{~Hz}) ; 4.81(\mathrm{t}, 2 \mathrm{H}, J=7.7 \mathrm{~Hz}) ; 2.85(\mathrm{dd}, 2 \mathrm{H}, J=7.7$, $12.3 \mathrm{~Hz}) .{ }^{13} \mathrm{C}$ NMR $(75 \mathrm{MHz}$, acetone-d 6 ) $\delta 146.7,146.1,140.6,126.6,126.0,122.1$, 53.7, 22.9. $\mathrm{MS}\left(\mathrm{ESI}^{+}\right) \mathrm{m} / \mathrm{z} 132\left(\mathrm{M}^{+}\right)$. Anal. Calcd. for $\mathrm{C}_{10} \mathrm{H}_{11} \mathrm{NSO}_{3} \mathrm{~F}_{3}: \mathrm{C}, 42.71 ; \mathrm{H}, 3.58$; N, 4.98; S, 11.40. Found: C, 42.40; H, 3.76; N, 4.85; S, 11.34 .

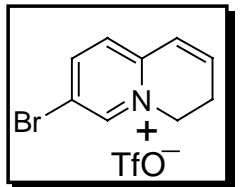

7-Bromo-3,4-dihydroquinolizinium triflate $\mathbf{( 3 b )}$. Following the general procedure, after stirring for $2 \mathrm{~h}, 72.0 \mathrm{mg}(80 \%)$ of $\mathbf{3 b}$ were 
obtained as a grey solid: mp $65-67^{\circ} \mathrm{C}\left(\mathrm{CH}_{2} \mathrm{Cl}_{2}\right.$ : $\left.\mathrm{Et}_{2} \mathrm{O}\right)$. IR ( $\left.\mathrm{NaCl}\right) 3069,1641,1504$, 1258, 1167, 1029, $857 \mathrm{~cm}^{-1} ;{ }^{1} \mathrm{H}$ NMR (300 MHz, acetone-d $\left.{ }_{6}\right) \delta 9.22(\mathrm{~s}, 1 \mathrm{H}) ; 8.75(\mathrm{dd}$, $1 \mathrm{H}, J=1.8,8.6 \mathrm{~Hz}) ; 7.97(\mathrm{~d}, 1 \mathrm{H}, J=8.6 \mathrm{~Hz}) ; 7.09-6.98(\mathrm{~m}, 2 \mathrm{H}) ; 4.94(\mathrm{t}, 2 \mathrm{H}, J=7.9$ $\mathrm{Hz}) ; 2.99-2.93$ (m, 2H). ${ }^{13} \mathrm{C}$ NMR (75 MHz, acetone-d $\left.{ }_{6}\right) \delta 149.3,147.7,147.4,141.7$, 127.7, 122.2, 119.9, 54.5, 32.2. MS (ESI $\left.{ }^{+}\right) \mathrm{m} / \mathrm{z} 210\left(\mathrm{M}^{+}\right), 212(\mathrm{M}+2)$. Anal. Calcd. for $\mathrm{C}_{10} \mathrm{H}_{9} \mathrm{NBrSO}_{3} \mathrm{~F}_{3}: \mathrm{C}, 33.35 ; \mathrm{H}, 2.52 ; \mathrm{N}, 3.89$. Found: C, 33.21; H, 2.42; N, 3.53.

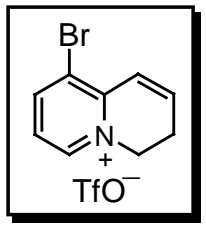

9-Bromo-3,4-dihydroquinolizinium triflate (3c). Following the general procedure, after stirring for $1 \mathrm{~h}, 57.6 \mathrm{mg}(80 \%)$ of $\mathbf{3 c}$ were obtained as a pale-brown solid: mp $145-147^{\circ} \mathrm{C}\left(\mathrm{CH}_{2} \mathrm{Cl}_{2}\right.$ : $\left.\mathrm{Et}_{2} \mathrm{O}\right)$. IR ( $\left.\mathrm{NaCl}\right) 3084,1632$, $1477,1262,1149,1029,784 \mathrm{~cm}^{-1} ;{ }^{1} \mathrm{H}$ NMR $\left(300 \mathrm{MHz}, \mathrm{CDCl}_{3}\right) \delta 9.03(\mathrm{~d}$, $1 \mathrm{H}, J=5.8 \mathrm{~Hz}) ; 8.86(\mathrm{~d}, 1 \mathrm{H}, J=8.2 \mathrm{~Hz}) ; 7.94(\mathrm{t}, 1 \mathrm{H}, J=8.1 \mathrm{~Hz}) ; 7.27-7.17(\mathrm{~m}, 2 \mathrm{H})$; $4.97(\mathrm{t}, 2 \mathrm{H}, J=7.5 \mathrm{~Hz}) ; 3.02-2.85(\mathrm{~m}, 2 \mathrm{H}) .{ }^{13} \mathrm{C}$ NMR $\left(75 \mathrm{MHz}\right.$, acetone-d $\left.{ }_{6}\right) \delta 150.1$, 147.2, 146.2, 143.8, 126.3, 121.7, 121.2, 55.4, 22.8. MS $\left(\mathrm{ESI}^{+}\right) \mathrm{m} / \mathrm{z} 211\left(\mathrm{M}^{+}\right) 213$ $(\mathrm{M}+2)$. Anal. Calcd. for $\mathrm{C}_{10} \mathrm{H}_{9} \mathrm{NBrSO}_{3} \mathrm{~F}_{3}$ : C, 33.35; H, 2.52; N, 3.89. Found: C, 33.12; $\mathrm{H}, 2.74 ; \mathrm{N}, 3.51$.

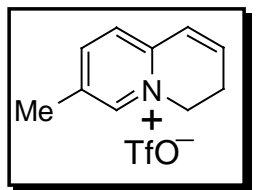

7-Methyl-3,4-dihydroquinolizinium triflate (3d). Following the general procedure, after stirring for $1 \mathrm{~h}, 48.4 \mathrm{mg}(82 \%)$ of $\mathbf{3 d}$ were obtained as a white powder: mp $94-96{ }^{\circ} \mathrm{C}\left(\mathrm{CH}_{2} \mathrm{Cl}_{2}\right.$ : $\left.\mathrm{Et}_{2} \mathrm{O}\right) . \mathrm{IR}(\mathrm{NaCl})$ 3048, 1643, 1531, 1265, 1152, 1032, $863 \mathrm{~cm}^{-1} ;{ }^{1} \mathrm{H}$ NMR (300 MHz, acetone-d 6 ) $\delta 8.80$ (s, $1 \mathrm{H}) ; 8.40(\mathrm{~d}, 1 \mathrm{H}, J=8.2 \mathrm{~Hz}) ; 7.88(\mathrm{~d}, 1 \mathrm{H}, J=8.2 \mathrm{~Hz}) ; 6.96-6.87(\mathrm{~m}, 2 \mathrm{H}) ; 4.83(\mathrm{t}$, $2 \mathrm{H}, J=7.9 \mathrm{~Hz}) ; 2.92-2.89(\mathrm{~m}, 2 \mathrm{H}) ; 2.53(\mathrm{~s}, 3 \mathrm{H}) .{ }^{13} \mathrm{C}$ NMR $\left(75 \mathrm{MHz}\right.$, acetone-d $\left.\mathrm{d}_{6}\right) \delta$ 147.5, 145.9, 139.6, 137.5, 126.3, 122.2, 54.0, 23.2, 18.0. MS (ESI $\left.{ }^{+}\right) \mathrm{m} / \mathrm{z} 146\left(\mathrm{M}^{+}\right)$. Anal. Calcd. for $\mathrm{C}_{11} \mathrm{H}_{12} \mathrm{NSO}_{3} \mathrm{~F}_{3}$ : C, 44.74; H, 4.10; N, 4.74; S, 10.86. Found: C, 44.56; H, 3.93; N, 4.48; S, 11.52 .

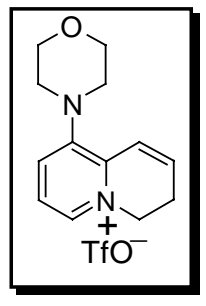

9-Morpholin-4-yl-3,4-dihydroquinolizinium triflate (3e). Following the general procedure, after stirring for $2 \mathrm{~h}, 62.2 \mathrm{mg}(85 \%)$ of $3 \mathrm{e}$ were obtained as a green oil: IR $(\mathrm{NaCl}) 3087,1636,1488,1261,1158,1030$, 
$786 \mathrm{~cm}^{-1} ;{ }^{1} \mathrm{H}$ NMR $\left(300 \mathrm{MHz}\right.$, acetone-d $\left.{ }_{6}\right) \delta 8.58(\mathrm{~d}, 1 \mathrm{H}, J=6.0 \mathrm{~Hz}) ; 8.25(\mathrm{~d}, 1 \mathrm{H}, J=$ $8.4 \mathrm{~Hz}) ; 7.89(\mathrm{dd}, 1 \mathrm{H}, J=6.0,8.4 \mathrm{~Hz}) ; 7.12-6.99$ (m, 2H); $4.84(\mathrm{t}, 2 \mathrm{H}, J=7.7 \mathrm{~Hz}) ; 3.85$ $(\mathrm{t}, 4 \mathrm{H}, J=4.6 \mathrm{~Hz}) ; 3.12(\mathrm{t}, 4 \mathrm{H}, J=4.6 \mathrm{~Hz}) ; 2.93-2.86(\mathrm{~m}, 2 \mathrm{H}) .{ }^{13} \mathrm{C} \mathrm{NMR}(75 \mathrm{MHz}$, acetone- $\left._{6}\right) \delta 148.8,143.2,139.2,139.0,135.4,125.6,118.9,66.4,53.9,52.2,22.1 . M S$ $\left(\mathrm{ESI}^{+}\right) \mathrm{m} / \mathrm{z} 217\left(\mathrm{M}^{+}\right)$. Anal. Calcd. for $\mathrm{C}_{14} \mathrm{H}_{17} \mathrm{~N}_{2} \mathrm{SO}_{4} \mathrm{~F}_{3}$ : C, 45.90; H, 4.68; N, 7.65. Found: C, 45.71; H, 4.75; N, 7.42; 8.64.

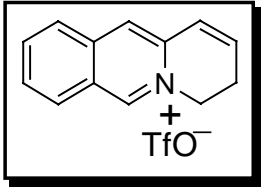

3,4-Dihydropyrido[1,2-b]isoquinolinium triflate (3f). Following the general procedure, after stirring for $3 \mathrm{~h}, 49.6 \mathrm{mg}$ (75\%) of $\mathbf{3 f}$ were obtained as a white powder: $\mathrm{mp} 105-107{ }^{\circ} \mathrm{C}\left(\mathrm{CH}_{2} \mathrm{Cl}_{2}: \mathrm{Et}_{2} \mathrm{O}\right)$. IR $(\mathrm{NaCl}) 3053,1652,1428,1261,1155,1029,755 \mathrm{~cm}^{-1} ;{ }^{1} \mathrm{H}$ NMR $\left(300 \mathrm{MHz}, \mathrm{CDCl}_{3}\right) \delta$ $10.13(\mathrm{~s}, 1 \mathrm{H}) ; 8.51(\mathrm{~d}, 1 \mathrm{H}, J=8.2 \mathrm{~Hz}) ; 8.07-7.98(\mathrm{~m}, 2 \mathrm{H}) ; 7.87-7.83(\mathrm{~m}, 2 \mathrm{H}) ; 6.77(\mathrm{~d}$, $2 \mathrm{H}, J=9.9 \mathrm{~Hz}) ; 6.70-6.63(\mathrm{~m}, 2 \mathrm{H}) ; 5.00(\mathrm{t}, 2 \mathrm{H}, J=7.1 \mathrm{~Hz}) ; 2.89-2.84(\mathrm{~m}, 2 \mathrm{H}) .{ }^{13} \mathrm{C}$ NMR (75 MHz, acetone- $\left.\mathrm{d}_{6}\right) \delta 151.7,139.4,137.6,135.8,131.3,131.2,127.9,127.2$, 123.3, 123.2, 122.4, 55.0, 23.2. MS $\left(\mathrm{ESI}^{+}\right) \mathrm{m} / \mathrm{z} 182\left(\mathrm{M}^{+}\right)$. Anal. Calcd. for $\mathrm{C}_{14} \mathrm{H}_{12} \mathrm{NSO}_{3} \mathrm{~F}_{3}$ : C, 50.75; H, 3.65; N, 4.23; S, 9.68. Found: $\mathrm{C}, 50.65 ; \mathrm{H}, 3.54 ; \mathrm{N}, 4.18$; S, 9.55 .

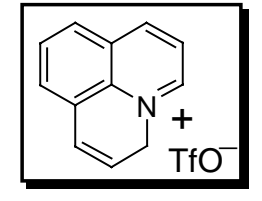

3H-Pyrido[3,2,1-ij]quinolinium triflate $(3 \mathrm{~g})$. Following the general procedure, stirring for $1.5 \mathrm{~h}, 50.0 \mathrm{mg}(79 \%)$ of $\mathbf{3 g}$ were obtained as a brown solid: $\mathrm{mp} 171-173{ }^{\circ} \mathrm{C}$ (Acetone: $\mathrm{Et}_{2} \mathrm{O}$ ). IR ( $\left.\mathrm{NaCl}\right) 1587,1266$, 1150, 1030, $844 \mathrm{~cm}^{-1} ;{ }^{1} \mathrm{H}$ NMR (300 MHz, acetone-d $\left.{ }_{6}\right) \delta 9.42(\mathrm{~d}, 1 \mathrm{H}, J=5.5 \mathrm{~Hz}) ; 9.20$ $(\mathrm{d}, 1 \mathrm{H}, J=8.2 \mathrm{~Hz}) ; 8.27-8.17(\mathrm{~m}, 2 \mathrm{H}) ; 7.91-7.88(\mathrm{~m}, 2 \mathrm{H}) ; 6.99(\mathrm{~d}, 1 \mathrm{H}, J=10.2 \mathrm{~Hz})$; 6.47-6.44 (m, 1H); 6.07 (s, 2H). ${ }^{13} \mathrm{C}$ NMR (75 MHz, acetone-d $\left.{ }_{6}\right) \delta$ 167.5, 149.1, 147.4, 131.7, 131.2, 130.9, 129.1, 126.5, 125.4, 123.7, 123.8, 56.9. MS (ESI $\left.{ }^{+}\right) \mathrm{m} / \mathrm{z} 167\left(\mathrm{M}^{+}\right)$. Anal. Calcd. for $\mathrm{C}_{12} \mathrm{H}_{8} \mathrm{NSO}_{3} \mathrm{~F}_{3}$ : C, 49.21; H, 3.18; N, 4.41; S, 10.11. Found: C, 49.11; H, 3.49; N, 4.17; S, 10.27.

\section{Ring-closing metathesis of salts 4. General procedure for cations $3 h$-j.}

To a solution of $5 \mathrm{mmol} \%$ ruthenium catalyst 2 (8.5mg, $0.01 \mathrm{mmol})$ in dry $\mathrm{CH}_{2} \mathrm{Cl}_{2}$ (35 $\mathrm{mL})$ was added under argon a solution of the corresponding salt $\mathbf{4 h}-\mathbf{j}(0.2 \mathrm{mmol})$ in dry $\mathrm{CH}_{2} \mathrm{Cl}_{2}(5 \mathrm{~mL}, 0.005 \mathrm{M})$. After stirring for $1-2 \mathrm{~h}$ at room temperature $(3 \mathbf{h}, \mathbf{i})$ or heating 
at $40{ }^{\circ} \mathrm{C}(\mathbf{3 j})$, the solvent was evaporated under reduced pressure and the residue was purified by flash chromatography on silica gel (eluent: $\mathrm{CH}_{2} \mathrm{Cl}_{2} / \mathrm{MeOH}$ 9.3:0.7) (3j) or by washing with $\mathrm{CH}_{2} \mathrm{Cl}_{2} / \mathrm{Et}_{2} \mathrm{O}$ (3h and $\mathbf{3 i}$ ).

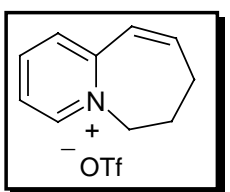

7,8-Dihydro-6H-pyrido[1,2-a]azepinylium triflate (3h). Following the general procedure, after stirring for $1.5 \mathrm{~h}, 51.9 \mathrm{mg}(88 \%)$ of $\mathbf{3 h}$ were obtained as grey solid: $\mathrm{mp} 97-98^{\circ} \mathrm{C}\left(\mathrm{CH}_{2} \mathrm{Cl}_{2}\right.$ : $\left.\mathrm{Et}_{2} \mathrm{O}\right)$. IR $(\mathrm{NaCl})$ 3094, 1647, 1460, 1259, 1147, 1031, $801 \mathrm{~cm}^{-1} ;{ }^{1} \mathrm{H}$ NMR (300 MHz, $\left.\mathrm{CDCl}_{3}\right) \delta 9.00$ $(\mathrm{d}, 1 \mathrm{H}, J=6.2 \mathrm{~Hz}) ; 8.57(\mathrm{td}, 1 \mathrm{H}, J=1.3,8.9 \mathrm{~Hz}) ; 8.12(\mathrm{~d}, 1 \mathrm{H}, J=8.0 \mathrm{~Hz}) ; 7.97(\mathrm{t}, 1 \mathrm{H}, J$ $=6.6 \mathrm{~Hz}) ; 6.93-6.81(\mathrm{~m}, 2 \mathrm{H}) ; 4.89(\mathrm{t}, 2 \mathrm{H}, J=4.6 \mathrm{~Hz}) ; 2.80-2.75(\mathrm{~m}, 2 \mathrm{H}) ; 2.47-2.39(\mathrm{~m}$, $2 \mathrm{H}) .{ }^{13} \mathrm{C}$ NMR $\left(75 \mathrm{MHz}\right.$, acetone- $\left.\mathrm{d}_{6}\right) \delta 148.5,146.3,146.0,139.5,131.6,126.4,122.7$, 62.4, 32.6, 28.0. MS (ESI $\left.{ }^{+}\right) \mathrm{m} / \mathrm{z} 146\left(\mathrm{M}^{+}\right)$. Anal. Calcd. for $\mathrm{C}_{11} \mathrm{H}_{12} \mathrm{NSO}_{3} \mathrm{~F}_{3}: \mathrm{C}, 44.74 ; \mathrm{H}$, 4.10; N, 4.74; S, 10.86. Found: C, 44.66; H, 3.97; N, 4.38; S, 10.74.

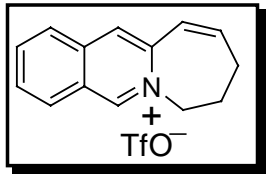

8,9-Dihydro-7H-azepino[1,2-b]isoquinolinium triflate (3i).

Following the general procedure, after stirring for $2 \mathrm{~h}, 64.8 \mathrm{mg}(94 \%)$ of $3 \mathbf{i}$ were obtained as a pale-brown powder: mp $156-158{ }^{\circ} \mathrm{C}$ $\left(\mathrm{CH}_{2} \mathrm{Cl}_{2}\right.$ : Et2O). IR (NaCl) 3039, 1652, 1523, 1260, 1154, 1030, $754 \mathrm{~cm}^{-1} ;{ }^{1} \mathrm{H}$ NMR $\left(200 \mathrm{MHz}\right.$, acetone- $\left.\mathrm{d}_{6}\right) \delta 10.03(\mathrm{~s}, 1 \mathrm{H}) ; 8.52-8.47(\mathrm{~m}, 2 \mathrm{H}) ; 8.30-8.17(\mathrm{~m}, 2 \mathrm{H}) ; 8.05(\mathrm{td}$, $1 \mathrm{H}, J=1.3,7.9 \mathrm{~Hz}) ; 6.66-6.55(\mathrm{~m}, 1 \mathrm{H}) ; 5.05(\mathrm{t}, 2 \mathrm{H}, J=4.6 \mathrm{~Hz}) ; 2.78-2.70(\mathrm{~m}, 2 \mathrm{H})$; 2.54-2.43 (m, 2H). ${ }^{13} \mathrm{C}$ NMR (75 MHz, CD $\left.3 \mathrm{OD}\right) \delta 151.3,143.6,142.3,139.7,138.2$, 132.2, 131.1, 128.9, 128.3, 127.7, 122.8, 62.6, 31.8, 27.8. MS $\left(\mathrm{ESI}^{+}\right) \mathrm{m} / \mathrm{z} 196\left(\mathrm{M}^{+}\right)$. Anal. Calcd. for $\mathrm{C}_{15} \mathrm{H}_{14} \mathrm{NSO}_{3} \mathrm{~F}_{3}: \mathrm{C}, 52.17 ; \mathrm{H}, 4.09 ; \mathrm{N}, 4.06 ; \mathrm{S}, 9.28$. Found: C, 51.99; H, $4.14 ; \mathrm{N}, 4.19 ; \mathrm{S}, 9.34$.

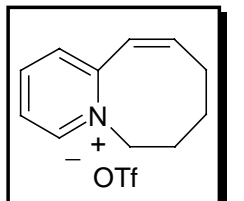

6,7,8,9-Tetrahydropyrido[1,2-a]azocinylium triflate $(3 \mathbf{j})$. Following the general procedure, after stirring for $1 \mathrm{~h}$ at $40^{\circ} \mathrm{C}, 33.4 \mathrm{mg}(54 \%)$ of $\mathbf{3 j}$ were obtained as a white solid: $85-86^{\circ} \mathrm{C}\left(\mathrm{CH}_{2} \mathrm{Cl}_{2}: \mathrm{Et}_{2} \mathrm{O}\right)$. IR $(\mathrm{NaCl})$ 2928, 1649, 1465, 1263, 1031, $638 \mathrm{~cm}^{-1} ;{ }^{1} \mathrm{H}$ NMR (300 MHz, acetone-d $\left.{ }_{6}\right) \delta 9.08(\mathrm{~d}, 1 \mathrm{H}$, $J=6.0 \mathrm{~Hz}) ; 8.62(\mathrm{t}, 1 \mathrm{H}, J=7.7 \mathrm{~Hz}) ; 8.14-8.09(\mathrm{~m}, 2 \mathrm{H}) ; 6.73-6.67(\mathrm{~m}, 2 \mathrm{H}) ; 4.92(\mathrm{t}, 2 \mathrm{H}$, $J=5.7 \mathrm{~Hz}) ; 2.60-2.55(\mathrm{~m}, 2 \mathrm{H}) ; 2.22-2.17(\mathrm{~m}, 2 \mathrm{H}) ; 1.59-1.51(\mathrm{~m}, 2 \mathrm{H}) .{ }^{13} \mathrm{C} \mathrm{NMR}(75$ MHz, acetone- $\left.\mathrm{d}_{6}\right) \delta 147.3,145.6,145.5,129.9,127.6,119.6,58.5,32.0,29.4,20.0 . \mathrm{MS}$ 
$\left(\mathrm{ESI}^{+}\right) \mathrm{m} / \mathrm{z} 160\left(\mathrm{M}^{+}\right)$. Anal. Calcd. for $\mathrm{C}_{12} \mathrm{H}_{14} \mathrm{NSO}_{3} \mathrm{~F}_{3}: \mathrm{C}, 46.60 ; \mathrm{H}, 4.56 ; \mathrm{N}, 4.53 ; \mathrm{S}$, 10.37. Found: C, 46.78; H, 4.25; N, 4.43; S, 10.65. 